\title{
Analyzing and Classifying Risks: a Case-Study in the Furniture Industry
}

\author{
Celina P. Leão ${ }^{10000-003-3725-5771]}$, Matilde A. Rodrigues ${ }^{\text {[000-0001-6175-6934] }}$ and Irene \\ Brito $^{3[000-0002-7075-3265]}$ \\ ${ }^{1}$ Algoritmi Centre, University of Minho, Guimarães, Portugal \\ ${ }^{2}$ Research Centre on Environment and Health, School of Health of Polytechnic Institute of \\ Porto, Porto, Portugal \\ ${ }^{3}$ Centre of Mathematics and Department of Mathematics and Applications, University of Mi- \\ nho, Guimarães, Portugal \\ cpl@dps.uminho.pt, mar@estsp.ipp.pt, ireneb@math.uminho.pt
}

\begin{abstract}
In this work we propose a methodology which permits the risk analysis and classification of occupational accidents in industrial settings. Data used in this study corresponds to accidents that occurred in the furniture industry in Portugal in 2010. A loss random variable is constructed in order to model the number of lost days implied by different contact modes of injuries in industry. The corresponding risk measures, such as Value-at-Risk, expected loss, loss variance and exceedance probabilities are determined in order to analyze and classify the contact modes according to their risk level, allowing the identification of the most problematic and the less problematic accident category in this industry. Contact with cutting, sharp, rough material was the most problematic whereas contact with electrical current, temperature, hazardous substance was the one with lower risk level.
\end{abstract}

Keywords: risk analysis, risk classification, value-at-risk, industry.

\section{Introduction}

Furniture industry is one of the most important sectors of activity in Portugal, in particular in north and center of the country, underpin a significant part of this region economy [1]. This sector is dominated by small and medium-sized enterprises (SMEs) [2]. Like other SMEs, also furniture industry face several challenges, such as competition, turnover, ageing workforce or limited resources (human, financial and technological) [3-7]. These companies have also more difficulties with risk assessment process [5,1]; as a consequence, poor work conditions are expected.

Rodrigues et al. [1] in a study involving 14 small and medium-sized Portuguese furniture industries, found that these enterprises face several problems related to the use of unsafe machines, unsafe behavior and manual work.

Considering this scenario, it is not surprising the high number of occupational accidents in furniture industries [1]. Understanding the key scenarios related to 
occupational accidents in the furniture sector, as the probability of resulting in some damages to workers (consequences), appears of paramount importance in order to identify critical areas of intervention and help both authorities and enterprises to support decision-making and define an effective risk management strategy.

To this end, risk analysis can provide important information about accident events in furniture industry. Risk analysis is a process that involves developing an understanding of the risk, providing an input to decision making process (ISO 31000:2009). However, because SMEs lack sufficient accident information at the company level, several researchers recommend the use public statistics to support decision-making process about the risks related to occupational accidents (see e.g. Rodrigues et al. [8], CarrilloCastrillo et al. [9], Jacinto \& Silva 10]).

The Value-at-Risk (VaR) is a risk measurement tool frequently used in the field of financial management and actuarial risk theory. VaR refers to a loss level that will not be exceeded for a certain confidence level, during a certain period of time [11]. However, it can bring important inputs also for the risk of occupational accidents. In fact, VaR was already previously used to describe accident risks in different sectors and activities, such as accidents in energy sector [11] or highway hazmat shipments [12]. In the present study, $\mathrm{VaR}$ principles are adopted to identify the most important accident scenarios in the furniture industries.

In the methodology that we are proposing, a loss variable is defined in order to model the number of lost work days, depending on the accidents' occurrence probability and estimated number of lost days. Furthermore, expected loss, variance of loss and the Central Limit Theorem are used in the risk analysis and classification.

\section{Risk Analysis}

We consider six contact modes of injury categories, denoted by $i=1, \ldots, 6$, which occurred in the furniture industry in Portugal in 2010 (see Table 1.).

Table 1. Contact mode of injury categories.

\begin{tabular}{ll}
\hline$i$ & Injury category \\
\hline 1 & Contact with electrical voltage, temperatures, hazardous substances \\
2 & Horizontal or vertical impact with or against a stationary object (the victim is in motion) \\
3 & Struck by object in motion collision \\
4 & Contact with cutting, sharp, rough material \\
5 & Entrapment, crouching, among others \\
6 & Physical constriction of the body, psychic embarrassment \\
\hline
\end{tabular}

Let $n$ be the total number of accidents and $n_{i}$ be the number of accidents of category $i$ for $i=1, \ldots, 6$. Note that $n=n_{1}+\cdots+n_{6}$. Let $b_{T_{i}}, i=1, \ldots, 6$, denote the total number of lost days due to accidents of category $i$. 
We define the following variables:

$$
b_{i}=\frac{b_{T_{i}}}{n_{i}}
$$

represents the estimated number of lost days due to an accident of category $i$ and

$$
p_{i}=\frac{n_{i}}{n}
$$

the occurrence probability of an accident of category $i$, where $i=1, \ldots, 6$.

Considering the observed values according to the dataset of the 2010 work accidents in the furniture industrial sector, provided by the Portuguese Office of Strategy and Planning (GEP), we obtain the following results listed in Table 2.

Table 2. Summary of results for each contact mode of injury.

\begin{tabular}{ccccc}
\hline$i$ & $n_{i}$ & $b_{T_{i}}$ & $b_{i}$ & $p_{i}$ \\
\hline 1 & 97 & 1135 & 11.70 & 0.02 \\
2 & 523 & 17457 & 33.38 & 0.12 \\
3 & 958 & 18082 & 18.87 & 0.22 \\
4 & 1406 & 53661 & 38.17 & 0.33 \\
5 & 331 & 13594 & 41.07 & 0.08 \\
6 & 998 & 27062 & 27.12 & 0.23 \\
\hline
\end{tabular}

One can observe that the accident category 4 has the highest occurrence. Considering the estimated number of lost days, it is accident category 5 which implies a higher number of lost days, however its occurrence probability is low, $p_{5}=0.08$, when e.g. compared with $p_{4}=0.33$.

In order to analyze the risks - lost days implied by each accident category - one must take into account the occurrence probability and the estimated number of lost days of each accident category. Therefore, we define the following loss random variable

$$
X_{i}=I_{i} b_{i},
$$

representing the lost days associated to an accident of category $i$, where $I_{i}$ is a Bernoulli $\left(p_{i}\right)$ distributed indicator random variable, with $p_{i}$ defined in (2), and $b_{i}$ is defined in (1). The expected loss and variance of the loss associated to an accident of category $i$ are calculated as follows

$$
\begin{gathered}
E\left[X_{i}\right]=b_{i} E\left[I_{i}\right]=b_{i} p_{i}, \\
\operatorname{Var}\left[X_{i}\right]=b_{i}^{2} \operatorname{Var}\left[I_{i}\right]=b_{i}^{2} p_{i}\left(1-p_{i}\right) .
\end{gathered}
$$

In order to classify the risks, we will also determine the probability of the loss random variable exceeding 7 days (one week) and 15 days (half a month) using the Central Limit Theorem: 


$$
P\left(X_{i}>\alpha\right) \approx 1-\Phi\left(\frac{\alpha-E\left[X_{i}\right]}{\sqrt{\operatorname{Var}\left[X_{i}\right]}}\right)
$$

where $\alpha=7$ and $\alpha=15$.

The Value-at-Risk ( $\mathrm{VaR})$ is a standard risk measure in actuarial risk theory used to evaluate the exposure to risk (see e.g. Klugman et al. [13]). Since in the present context the loss is associated with an amount of capital, the VaR is useful to predict the amount needed for each risk that exceeds the lost amount with a high degree of certainty. The $\mathrm{VaR}$ of a loss random variable $X$ at the $100 \mathrm{p} \%$ level, denoted $\operatorname{Var}_{p}(X)$ is the $100 \mathrm{p}$ percentile (or quantile) of the distribution of $X$. We write $\operatorname{Var}_{p}\left(X_{i}\right)$ as the value of $\pi_{p}$ satisfying

$$
P\left(X_{i}<\pi_{p}\right)=p
$$

In the present case we will determine the $95 \%$ quantile, setting $p=0.95$ in (7). Calculating (4), (5), (6) and (7) for the loss corresponding to the six contact modes of injury categories, we obtain the results listed in Table 3.

Table 3. Expected loss, variance, probabilities and VaR for each injury category.

\begin{tabular}{cccccc}
\hline$i$ & $E\left[X_{i}\right]$ & $\operatorname{Var}\left[X_{i}\right]$ & $P\left(X_{i}>7\right)$ & $P\left(X_{i}>15\right)$ & $\pi_{0.95}$ \\
\hline 1 & 0.26 & 3.01 & 0.00 & 0.00 & 3.12 \\
2 & 4.05 & 118.72 & 0.39 & 0.16 & 21.97 \\
3 & 4.19 & 61.55 & 0.36 & 0.08 & 17.10 \\
4 & 12.44 & 320.05 & 0.62 & 0.44 & 41.87 \\
5 & 3.15 & 119.51 & 0.36 & 0.14 & 21.14 \\
6 & 6.27 & 130.77 & 0.47 & 0.22 & 25.09 \\
\hline
\end{tabular}

\section{$3 \quad$ Analysis and Interpretation of Results}

From the results summarized in Table 3, several findings emerge and will be presented below.

The accident category which has the highest expected loss is accident category 4 with 12.44 days, whereas accident category 1 has the lowest expected loss: 0.26 days.

Accident category 4 has a considerable probability of exceeding 15 lost days: $P\left(X_{4}>15\right)=0.44$. For accident category 6 the corresponding probability is half of this value: $P\left(X_{6}>15\right)=0.22$, and for accident categories 1 and 3 it is approximately null.

Considering a loss of more than 7 days, the highest probability is attained again with accident category 4 , namely $P\left(X_{4}>7\right)=0.62$, followed by accident category 6 , for which $P\left(X_{6}>7\right)=0.47$. For accident category 1 , we can say that this probability is approximately null. For the contact mode of injury category 2 and 3 considering a loss of more than 7 days is approximately the same $\left(P\left(X_{2}>7\right)=0.39=P\left(X_{3}>7\right)=\right.$ 
$0.36)$, but this reduce by half when considering a loss of more than 15 days $\left(P\left(X_{2}>\right.\right.$ $15)=0.16$ and $\left.P\left(X_{3}>15\right)=0.08\right)$.

The Values-at-Risk, $\pi_{0.95}$, in the last column of Table 3, can be interpreted as follows. For accident category 4 , since $\pi_{0.95}=41.87$, there is a $95 \%$ of certainty, that the number of lost days will be less than 41.87 days, or in other words, there is a $5 \%$ chance of exceeding this number of lost days. Accident category 1 has the lowest VaR, $\pi_{0.95}=$ 3.12. This means that the risk of exceeding 3.12 lost days with accident category 1 is $5 \%$, or, with a probability of 0.95 the number of lost days will be less than 3.12 .

Note that accident category 4 has the highest variance, $\operatorname{Var}\left[X_{4}\right]=320.05$, thus this also indicates that this category of accident is riskier in the sense that it can lead to extremer values of lost days, i.e. to numbers of lost days which are very higher than the expected number of lost days.

Taking into account the previous results and analysis, we can classify the accident categories and order them according to their risk level. From the results given in Table 3 , we obtain the ordering in Table 4, where the categories are ordered from the left to the right, from the highest to the lowest risk level.

Table 4. Ordered injury categories.

\begin{tabular}{ccccccc}
\hline$E\left[X_{i}\right]$ & 4 & 6 & 3 & 2 & 5 & 1 \\
$\operatorname{Var}\left[X_{i}\right]$ & 4 & 6 & 5 & 2 & 3 & 1 \\
$P\left(X_{i}>7\right)$ & 4 & 6 & 2 & $3(5)$ & $5(3)$ & 1 \\
$P\left(X_{i}>15\right)$ & 4 & 6 & 5 & 2 & 3 & 1 \\
$\pi_{0.95}$ & 4 & 6 & 2 & 5 & 3 & 1 \\
\hline
\end{tabular}

We conclude that contact mode of injury 4 (contact with cutting, sharp, rough material) is the most problematic one for the industry, followed by contact mode of injury 6 (physical constriction of the body, psychic embarrassment), whereas contact mode of injury 1 (contact with electrical current, temperature, hazardous substance) is the one with lower risk level. As for injury categories 2 (crushing in vertical/horizontal movement on/against an immovable object (moving victim)), 3 (blow by moving object, collision) and 5 (entrapment, crouching, among others), which have an intermediate risk level, we can say that in terms of expected number of lost days, contact mode of injury 3 leads to a higher number of lost days, followed by categories 2 and 5 .

However, considering the variance and the probability of exceeding more than 15 lost days, the results indicate that contact mode of injury 5 is more problematic, followed by injury categories 2 and 3 . The probability of exceeding more than 7 days is higher for category 2; for categories 3 and 5 this probability is equal. Finally, taking into account the VaR, contact mode of injury 2 is riskier than 5 and both are riskier than contact mode of injury 3 .

\section{Conclusions}

In this work a methodology which permits the risk analysis and classification of occupational accidents in industrial settings is proposed. The Portuguese furniture industry 
accounts for a significant part of the economy, however, the figures for accidents at work remain high. It is therefore important to analyze accident data for future measures to prevent accidents.

A loss random variable is constructed in order to model the number of lost days implied by different contact modes of injuries obtained in this industry. The corresponding risk measures, such as VaR, expected loss, loss variance and exceedance probabilities are determined in order to analyze and classify the contact modes according to their risk level.

Our contribution lies in identification of the most problematic and, at the other end, the less problematic, accident category in this industry.

In accordance with the risk measures considered different results were obtained. Contact with cutting, sharp, rough material (contact mode of injury 4) is the most problematic contact mode injury obtained for the furniture industry. On the other hand, with lower risk level the contact with electrical current, temperature, hazardous substance (contact mode of injury 1). Considering the contact modes with intermediate risk level and the probability of exceeding more than 15 lost days, the results indicate that entrapment, crouching, among others (contact mode of injury 5) is the more problematic, and based on the VaR, horizontal or vertical impact with or against a stationary object (contact mode of injury 2) is the most risky contact mode of injury.

Future work will be aimed at addressing the same methodology with more current data and to analyze the development of the incidence of accidents.

\section{Acknowledgements}

The authors acknowledge support from FCT - Fundação para a Ciência e Tecnologia, within the Projects Scopes: UID/MAT/00013/2013 and UID/CEC/00319/2019.

\section{References}

1. Rodrigues, M.A., Arezes, P., Leão, C.P.: Characterization of the Portuguese Furniture Industry's Safety Performance and Monitoring Tools. In Arezes, P.M.F.M., Carvalho, P.V.R. (eds.). Ergonomics and Human Factors in Safety Management. Chapter 5, pp. 93-109. CRC Press Taylors and Francis, London (2016).

2. Eurostat (2013) Homepage, Archive:Manufacture of furniture statistics - NACE Rev. 2, https://ec.europa.eu/eurostat/statistics, last update 2013/04.

3. EU (European Commision) Homepage, Furniture industry, https://ec.europa.eu/growth/sectors/raw-materials/industries/forestbased/furniture_en, last accessed 13/11/2018.

4. Fabiano, B., Currò, F., Pastorino, R.: A study of the relationship between occupational injuries and firm seize and type in the Italian industry. Safety Science 42, 587-600 (2004).

5. Sørensen, O.H., Hasle, P., Bach, E.: Working in small enterprises - Is there a special risk? Safety Science 45, 1044-1059 (2007). 
6. Micheli, G., Cagno, E.: Dealing with SMEs as a whole in OSH issues: Warnings from empirical evidence. Safety Science, 48, $729-733$ (2010).

7. Rodrigues, M.A., Arezes, P., Leão, C.P.: Risk assessment: getting the 'big picture'. In Boustras, G., Guldenmund, F. (Eds). Handbook of Safety Management in SME's. Chapter 2. Taylor and Francis Group, London (2017).

8. Rodrigues, M.A., Arezes, P., Leão, C.P. Defining risk acceptance criteria in occupational settings: A case study in the furniture industrial sector. Safety Science, 80, 288295 (2015).

9. Carrillo-Castrillo, J.A., Rubio-Romero, J.C., Guadix, J., Onieva, L.: Risk assessment of maintenance operations: the analysis of performing task and accident mechanism. International Journal of Injury Control and Safety Promotion, 22 (3), 267-277 (2015).

10. Jacinto, C., Silva, C.: A semi-quantitative assessment of occupational risks using bowtie representation. Safety Science, 48(8), 973-979 (2010).

11. Spada, M., Paraschiv, F., Burgherr, P.: A comparison of risk measures for accidents in the energy sector and their implications on decision-making strategies. Energy 154, 277-288 (2018).

12. Hosseini, S.D., Verma, M.: A Value-at-Risk (VAR) approach to routing rail hazmat shipments. Transportation Research Part D 54, 191-211 (2017).

13. Klugman, S., Panjer, H., Willmot, G.: Loss Models. 4th edn. Wiley (2012). 